\title{
Structural Optimization of Thin-Walled Tubular Structures for Progressive Collapse Using Hybrid Cellular Automaton with a Prescribed Response Field
}

\author{
Homero Valladares, Joel Najmon, Andres Tovar
}

\begin{abstract}
The design optimization of thin-walled tubular structures is of relevance in the automotive industry due to their low cost, ease of manufacturing and installation, and high-energy absorption efficiency. This study presents a methodology to design thin-walled tubular structures for crashworthiness applications. During an impact, thinwalled tubular structures may exhibit progressive collapse/buckling, global collapse/buckling, or mixed collapse/buckling. From a crashworthiness standpoint, the most desirable collapse mode is progressive collapse due to its high-energy absorption efficiency, stable deformation, and low peak crush force (PCF). In the automotive industry, thin-walled components have complex structural geometries. These complexities and the several loading conditions present in a crash reduce the possibility of progressive collapse. The Hybrid Cellular Automata (HCA) method has shown to be an efficient continuum-based approach in crashworthiness design. All the current implementations of the HCA method use a scalar set point to design structures with a uniform distribution of a field variable, e.g., stress, strain, internal energy density (IED), mutual potential energy. For example, using IED and mutual potential energy as the field variable result in high stiffness and progressive collapsing structures, respectively. This paper presents a modified version of the HCA method to design thin-walled structures that collapse progressively. In this methodology, the set point has two components, a prescribed response field, which promotes progressive collapse, and a variable offset value, which satisfies the mass constraint. The numerical examples show that this modified HCA method is capable of finding material distributions that exhibit progressive collapse, resulting in significant improvement in specific energy absorption (SEA) with relatively little change in the PCF.
\end{abstract}

\section{Introduction}

The number of car accidents is an ever-increasing event due to the permanent demand for mass transportation. Within this context, researchers of the automotive industry continuously develop methodologies to improve the crash behavior of their vehicular structures to reduce the risk of injuries or death of the passengers. Thinwalled tubular components are widely used to manufacture structures with the ability to absorb the kinetic energy of the impact in a controllable and predictable manner [1]. The constitutive material, structural geometry, and loading conditions are the principal factors that determine the deformation mechanisms that absorb the kinetic energy of the impact. For example, metallic components absorb the kinetic energy through plastic deformation, while composite laminate components dissipate energy through several failure mechanisms that include matrix cracking, fiber fracture, delamination, frond formation, and bending [2].

The crush behavior of metallic thin-walled tubular structures has been well studied in the last decades since the pioneering work of Pugsley and Macaulay [3], and Alexander [4] in the 1960s. They experimentally studied the collapse of cylindrical tubes under quasistatic axial loading and developed theoretical models that include several features of the crumpling process. Later, Wierzbicki and Abramowicz presented a theory assuming rigid plasticity and kinematic continuity to study planar surface structures such as boxes beams, columns, and plate intersections [5]. Abramowicz and Jones modified this kinematic approach to include strain rate sensitivity of the material, in order to predict the collapse of circular and square tubes under static and dynamic axial loads [6-8]. The advancement in computing technologies has enabled the development of numerical implementations of the finite element method to study the crush behavior of thin-walled components [9-11].

Multiple numerical and experimental studies have shown that the dominant collapse modes of metallic thin-walled structures under axial loading are global, progressive, and mixed collapse. Mixed collapse initially starts with progressive collapse and ends in global collapse [1, 12, 13]. These collapse modes can occur at low (e.g., dynamic progressive collapse) or high (e.g., dynamic plastic collapse) impact velocities, however at high impact velocities, inertia effects are significant $[1,8,12]$. Progressive collapse is the most efficient mode for crashworthiness purposes [13]. During a progressive collapse, the plastic deformation of large amounts of material is used to absorb the kinetic energy of the impact. In general, thin-walled structures are designed so that the folding starts at the end closer to the impact and progresses along the structure towards the other end [14]. Researchers have explored several alternatives to promote progressive collapse in thin-walled components. The most popular solutions are the modification of the geometrical shape, the use of collapse initiators, and the implementation of filler materials. Other non-conventional methods include the use of multi-cell sections and functionally graded structures $[12,13]$.

The improvement in computational technologies, design optimization methods, and finite element analysis (FEA) formulations have propelled the design optimization of thin-walled structures for crashworthiness. FEA and optimization techniques find designs that maximize or minimize a given objective function satisfying certain

This is the author's manuscript of the article published in final edited form as:

Valladares, H., Najmon, J., \& Tovar, A. (2019). Structural Optimization of Thin-Walled Tubular Structures for Progressive Collapse Using Hybrid Cellular Automaton with a Prescribed Response Field (SAE Technical Paper No. 2019-01-0837). SAE International.

https://doi.org/10.4271/2019-01-0837 
functional and geometrical constraints. One of the most innovative optimization techniques for the design of thin-walled structures is topology optimization. This methodology finds optimal distribution of material along the design space in order to improve the crashworthiness of the component $[15,16]$. Gradient-based topology optimization techniques require the simplification of the complex interactions in the dynamic impact problem. The contact, geometrical, and material non-linearities limit the analytical evaluation of sensitivities and require an infeasible computational cost if they are evaluated numerically. A solution to this problem is the use of nongradient based optimization techniques like the Hybrid Cellular Automaton (HCA) method [17]. This makes the HCA method an efficient technique for the design of components for crashworthiness. The HCA method is a continuum-based approach that aims to find designs with a uniform distribution of a field variable. A set point drives the field variables to a common value by redistributing the material along the whole design domain. The HCA method finds designs with high stiffness and energy absorbing capabilities when the field variable is the IED [18]. Another version of the HCA method use the concept of compliant mechanisms to design thin-walled structures that collapse progressively. In this implementation, the HCA method finds designs with a uniform distribution of the mutual potential energy $[14,19]$.

In this work, we propose an extension of the HCA method that includes a prescribed response field as one of the components of the set point in order to promote progressive collapse. This modified HCA method is capable of designing progressively collapsing thin-walled tubular structures resulting in significant improvement in SEA with relatively little increase in the PCF.

\section{Topometry Optimization.}

Topometry optimization is an element-based topology optimization process whose design variables are the thickness of each shell element. The optimization process systematically changes the thickness distribution within the design domain to minimize or maximize an objective function.

The standard optimization problem is the minimization of an objective function subjected to one or more constraint functions, and bounds in the design variable. Common objectives of a topology optimization problem are the minimization of structural responses such as compliance, von Mises stress, or eigenfrequencies. A common constraint in the topology optimization problem is imposed on the mass or volume $(\mathrm{V})$ of the design.

$$
\begin{array}{lc}
\text { find } & \substack{\mathbf{x} \\
\min } \\
\text { s.t. } & \sum_{\mathrm{i}=1}^{\mathrm{N}} \mathrm{v}(\mathbf{x}) \\
& \mathbf{x} \in \chi, \quad \chi=\left\{\mathbf{x} \in \mathbb{R}^{n}: \mathrm{x}_{\text {min }} \leq \mathrm{x}_{\mathrm{i}} \leq \mathrm{x}_{\max }\right\},
\end{array}
$$

where $\mathbf{x}$ is the vector of design variables, $\mathbf{v}$ is a function for the elemental mass or volume, $\mathrm{N}$ is the number of elements in the design domain, and $x_{\min }$ and $x_{\max }$ are the bounds of the design variables [18].

\section{Design Methodology}

The HCA method is a computational approach to generate optimal topologies. Without explicitly being an optimization technique, the HCA method continuously improves the structural response of a Page 2 of 10 design by using local rules. The HCA method combines the cellular automaton (CA) paradigm with FEA $[17,18]$.

CA is a discrete model that consists of a grid of cells where the state of each cell is defined by a finite dimensional array. In the HCA method, the state of a CA, $\boldsymbol{\beta}_{\mathbf{i}}^{(\mathrm{k})}$, at discrete position i and iteration $\mathrm{k}$, corresponds to the design variable $\mathrm{x}_{\mathrm{i}}^{(\mathrm{k})}$ (e.g., density, thickness) and a field variable $S_{i}^{(k)}$ (e.g., stress, strain, IED or mutual potential energy or a function of these quantities) and is given by

$$
\boldsymbol{\beta}_{\mathrm{i}}^{(\mathbf{k})}=\left\{\begin{array}{l}
\mathrm{S}_{\mathrm{i}}^{(\mathrm{k})} \\
\mathrm{x}_{\mathrm{i}}^{(\mathrm{k})}
\end{array}\right\}
$$

Correspondence between the FEA mesh and the grid of CA grid is generally preferred but not required. Current HCA implementations attempt a uniform distribution of a field variable by redistributing the material along the whole design domain. With this purpose, all the field variables are driven to a common value called the set point $S_{i}^{*}$ [18]. The concept of obtaining optimal designs through uniform distribution of a field variable is similar to the fully stressed design approach, where the material is distributed over the whole design domain so that all the elements equally contribute to the structure's performance.

In this work, the set point $\mathbf{S}_{\mathbf{i}}^{*}$ is composed by two components. The first component is a prescribed field $\mathbf{S}_{\text {prescribed }}$ that resembles the response field of a design that progressively collapses. The second component is an offset value $\mathbf{c}$ that varies at each iteration to satisfy the mass constraint. This formulation of the set point is different from traditional HCA, which uses a common value for the entire design domain.

\section{Prescribed Field Variable}

The crucial aspect of the proposed methodology is the implementation of a prescribed field component in the set point of the HCA method. By prescribing an adequate response field, this modified HCA version finds designs with high-energy absorption and structural integrity. The prescribed set point $\mathrm{S}^{*}$ is defined as,

$$
\mathbf{S}^{*}=\mathrm{w} * \mathbf{S}_{\text {prescribed }}+\mathbf{c}
$$

where $\mathbf{S}_{\text {prescribed }}$ is the prescribed response field, w is a weight factor that controls the contribution of the prescribed field and $\mathbf{c}$ is an offset vector of value $c$, that is used to satisfy the mass constraint. At each iteration, $\mathrm{c}$ is determined using the bisection method. In this modified version of the HCA method, the set point is no longer a unique value for the whole design space, but rather an $\mathrm{N}$-dimensional quantity that promotes progressive collapse.

Since the purpose of this work is the design of thin-walled structures for progressive collapse, the prescribed set point is specified as the normalized IED field $\mathbf{S}_{\text {prescribed }}$ of structures that progressively collapse. Figure 1 is an example of a prescribed response field for progressive collapse. 


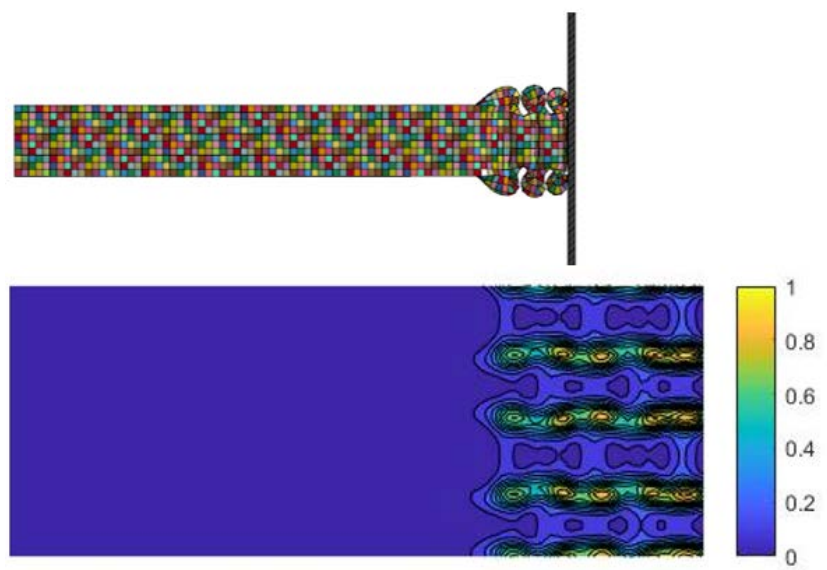

Figure 1. Prescribed response field that corresponds to the normalized IED at $500 \mathrm{~ms}$ of a $3 \mathrm{~mm}$ square tube impacted by a rigid wall with a velocity of $5 \mathrm{~m} / \mathrm{s}$.

\section{Normalized Field Variable}

In order to perform the material distribution, the final state $\bar{S}_{i}$ of a CA given by

$$
\bar{S}_{i}=\frac{1}{\widehat{N}+1} \sum_{j=0}^{\widehat{N}} S_{j}
$$

is determined. The final state of a cell takes into account the field information of itself $S_{0}$ and the field information $S_{j}$ of the $\widehat{N}$ cells in its neighborhood. The final state is an averaging scheme that works as a filtering technique that reduces checkerboarding patterns. Once the final states of all the cells have been determined, a normalization operation,

$$
\overline{\mathbf{S}}_{\text {norm }}=\frac{\overline{\mathbf{S}}-\min (\overline{\mathbf{S}})}{\max (\overline{\mathbf{S}})-\min (\overline{\mathbf{S}})}
$$

is applied to the entire design domain. This normalization is required to enable the comparison between the response field and the set point.

\section{Control Based Rules}

In the HCA method, control based rules regulate the distribution of the material along the whole design domain. This study uses a PID controller, for which the change in the design variable $\Delta \mathrm{x}_{\mathrm{i}}^{(\mathrm{k})}$ of the $\mathrm{i}^{\text {th }}$ element $\mathrm{x}_{\mathrm{i}}$ at the $\mathrm{k}^{\text {th }}$ iteration is expressed as [20],

$$
\Delta \mathrm{x}_{\mathrm{i}}^{(\mathrm{k})}=\mathrm{K}_{\mathrm{p}} \mathrm{e}_{\mathrm{i}}^{(\mathrm{k})}+\mathrm{K}_{\mathrm{i}} \int_{\mathrm{k}-2}^{\mathrm{k}} \mathrm{e}_{\mathrm{i}}^{(\mathrm{k})} \mathrm{dk}+\mathrm{K}_{\mathrm{d}} \Delta \mathrm{e}_{\mathrm{i}}^{(\mathrm{k})}
$$

where $\mathrm{K}_{\mathrm{p}}, \mathrm{K}_{\mathrm{i}}$, and $\mathrm{K}_{\mathrm{d}}$ correspond to the proportional, integral and derivative control gains, respectively and $e_{i}^{(k)}$ is the error between the final state of the CA and the set point given by

$$
\mathrm{e}_{\mathrm{i}}^{(\mathrm{k})}=\overline{\mathrm{S}}_{\mathrm{norm}(\mathrm{i})}-\mathrm{S}_{\mathrm{i}}^{*}
$$

It is important to note that the set point $\mathbf{S}^{*}$ does not necessarily remain constant through the optimization. As stated before, the set point is the Page 3 of 10 summation of the spatial distribution of prescribed values $\mathbf{S}_{\text {prescribed }}$ that promotes progressive collapse and an offset value $\mathbf{c}$ used to satisfy the mass constraint at each iteration.

In this study, the error $\mathbf{e}^{(\mathbf{k})}$ measures the difference between the IED distribution of the current design and the set point that represents the desired IED distribution. The control rule uses $\mathbf{e}^{(\mathbf{k})}$ to distribute the material. At an iteration $\mathrm{k}$, the $\mathrm{i}^{\text {th }} \mathrm{CA}$ is in equilibrium when $\mathrm{e}_{\mathrm{i}}^{(\mathrm{k})}=0$. If this condition is not satisfied, material is redistributed along the whole design domain. In order to avoid numerical instabilities, a limit $\Delta \mathrm{x}_{\text {lim }}$ over the minimum and maximum change of the design variable is applied. The design variable change $\Delta \mathrm{x}_{\mathrm{i}}^{(\mathrm{k})}$ at each iteration is,

$$
\Delta \mathrm{x}_{\mathrm{i}}^{(\mathrm{k})}=\max \left\{-\Delta \mathrm{x}_{\mathrm{lim}}, \min \left\{\Delta \mathrm{x}_{\mathrm{i}}^{(\mathrm{k})}, \Delta \mathrm{x}_{\mathrm{lim}}\right\}\right\}
$$

The new value of the design variable is

$$
\mathbf{x}_{\mathbf{i}}^{(\mathbf{k}+\mathbf{1})}=\mathbf{x}_{\mathbf{i}}^{(\mathbf{k})}+\Delta \mathbf{x}_{\mathbf{i}}^{(\mathbf{k})}
$$

\section{Mass Constraint}

By itself, the updating rule for $\Delta \mathrm{x}_{\mathrm{i}}^{(\mathrm{k})}$, does not impose any constraint in the mass (or volume) on the updated design $\mathbf{x}^{(\mathrm{k}+1)}$. To enforce a mass constraint, an appropriate value for the offset value $c$ is determined in a secondary inner loop based on the difference in mass of the updated design and the desired mass. The offset value $\mathrm{c}$ adjusts the set point so that after applying the updating rules for $\Delta \mathbf{x}^{(\mathbf{k})}$, the final design $\mathbf{x}^{(\mathrm{k}+1)}$ satisfies the mass constraint. 


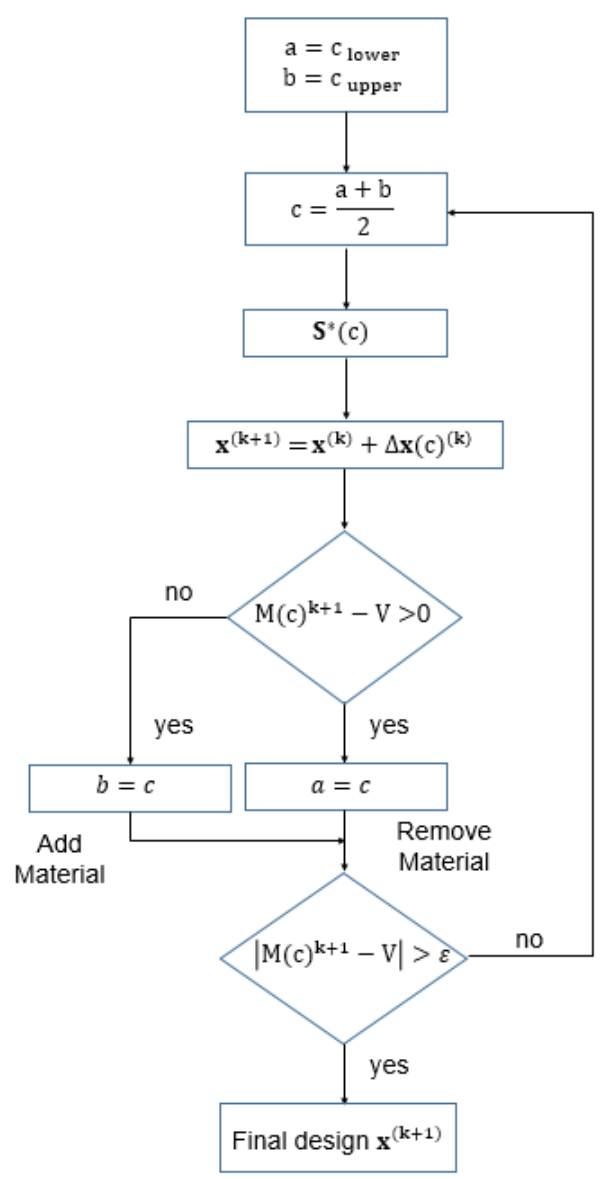

Figure 2. Inner loop (bisection method) flowchart used to determine offset value

The inner loop follows the bisection method scheme indicated at Figure 2. At each outer iteration $\mathrm{k}$, lower $\mathrm{c}_{\text {lower }}$ and upper $\mathrm{c}_{\text {upper }}$ bounds on $\mathrm{c}$ are defined. The offset value is the mean of the lower and upper moving limits $\mathrm{a}$ and $\mathrm{b}$. These limits are updated depending on the difference between the updated mass fraction $\mathrm{M}(\mathrm{c})^{(\mathrm{k}+1)}$ and the desired mass constraint V. If the difference is positive, the lower moving limit a is updated with the current value of $c$. This operation increases the set point $\mathbf{S}^{*}$ (c) in order to remove material from the design. If material should be added to the design the set point is lowered by updating the upper moving limit $\mathrm{b}$ as $\mathrm{c}$. This process is repeated iteratively until the difference between the current mass fraction and the mass constraint is within a tolerance value $\varepsilon$.

The optimization methodology of this paper, illustrated in Figure 3, is described as follows:

1. Initial design: Define the design domain, material properties, loading conditions, and initial thickness distribution.

2. Crash analysis: Determine the $\operatorname{IED} \mathrm{U}_{\mathrm{i}}^{(\mathrm{k})}$ at each $\mathrm{i}^{\text {th }}$ element using FEA.

3. Update material distribution: Use the updating rules and the inner optimization loop to determine the new design $\mathbf{x}^{(\mathrm{k}+1)}$.

4. Convergence test: Determine if convergence criteria have been satisfied. Otherwise, the iterative process is executed from step 2.

Page 4 of 10

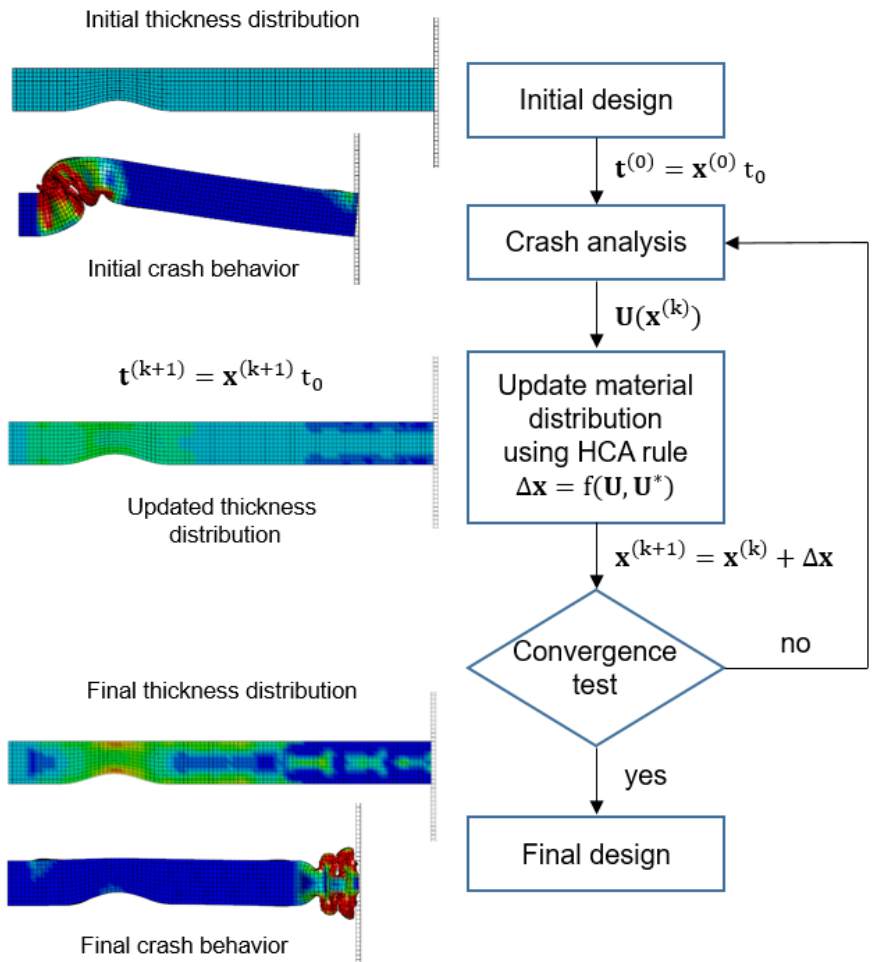

Figure 3. HCA methodology with prescribed set point for the design of thinwalled structures.

\section{Numerical Model}

The explicit nonlinear finite element code LS-DYNA is used to perform the dynamic simulations of this study. The thin-walled structures are modeled using a linear elastic, piecewise linear plastic material model (*MAT_24). The tube models are made of steel whose material properties are included in Table 1. Multiple investigations have shown an acceptable agreement between plane stress shell-based models and experimental results when studying thin-walled tubular structures under compressive loads. A plane stress shell element formulation with four in-plane integration points (ELEFORM $=16$ ) is used in this work. This is an efficient formulation that controls hourglass effects by implementing strain interpolants that alleviate locking and enhance in-plane bending behavior [21].

The thin-walled tube designs are impacted by a rigid plate with a prescribed velocity of $5 \mathrm{~m} / \mathrm{s}$. The contact between the tubes and the rigid plate is modeled with a friction coefficient of 0.3 to account for sliding movement. A single surface contact algorithm with a friction coefficient of 0.1 is used to account for the contact between sections of the tube during the folding process. 


\begin{tabular}{|c|c|}
\hline Property & Value \\
\hline Density & $7800 \mathrm{~kg} / \mathrm{m}^{3}$ \\
\hline Elastic Modulus & $207 \mathrm{GPa}$ \\
\hline Poisson's Ratio & 0.29 \\
\hline Yield Stress & $253 \mathrm{MPa}$ \\
\hline Effective plastic strain & Effective stress (MPa) \\
\hline 0.000 & 253 \\
\hline 0.048 & 367 \\
\hline 0.108 & 420 \\
\hline 0.148 & 442 \\
\hline 0.208 & 468 \\
\hline 0.407 & 524 \\
\hline 0.607 & 561 \\
\hline 0.987 & 608 \\
\hline
\end{tabular}

\section{Numerical Examples}

The proposed methodology is used to design two thin-walled tubular structures; a square tube and an S-rail. Both models have a geometric imperfection (i.e., dent) near their fixed edge. In order to promote progressive collapse, the prescribed response field of the HCA algorithm should correspond to a design that has undergone progressive collapse. In this study, the prescribed field is the IED of a uniform thickness square tube of $3 \mathrm{~mm}$ impacted by a rigid wall with a prescribed velocity of $5 \mathrm{~m} / \mathrm{s}$. The IED values are taken at a time of $500 \mathrm{~ms}$ (Figure 1). All the numerical examples are solved using 100 iterations of the modified HCA method. An expression that represents the global change of the design variable in the last three iterations is the index that measures the convergence of the algorithm as follows,

$$
\text { Convergence }=\frac{1}{2}\left(\sum_{\mathrm{i}=1}^{\mathrm{N}}\left|\mathrm{x}_{\mathrm{i}}^{(\mathrm{k}-2)}-\mathrm{x}_{\mathrm{i}}^{(\mathrm{k}-1)}\right|+\left|\mathrm{x}_{\mathrm{i}}^{(\mathrm{k}-1)}-\mathrm{x}_{\mathrm{i}}^{(\mathrm{k})}\right|\right)
$$

where $\mathrm{x}_{\mathrm{i}}^{(\mathrm{k}-2)}, \mathrm{x}_{\mathrm{i}}^{(\mathrm{k}-1)}$ and $\mathrm{x}_{\mathrm{i}}^{(\mathrm{k})}$ are the thickness values of the $\mathrm{i}^{\text {th }}$ element at the last three iterations.

\section{Square Tube}

The first example is a straight square tube with a geometric imperfection. The finite element model has a mesh size of $40 \times 101$ with an average element size of $0.01 \times 0.01 \mathrm{~m}$. The square tube's side length is $0.1 \mathrm{~m}$. In the FEA model, the nodes of one end of the tube are fixed, while the other end is axially impacted by a rigid plate with a prescribed constant velocity of $5 \mathrm{~m} / \mathrm{s}$ (Figure 4). During the optimization process, the thickness of each shell element can range from 3 to $10 \mathrm{~mm}$. The optimized designs must satisfy a mass constraint of 0.5 . The weight of the prescribed field $w$ is 0.5 and the simulation time is $500 \mathrm{~ms}$.

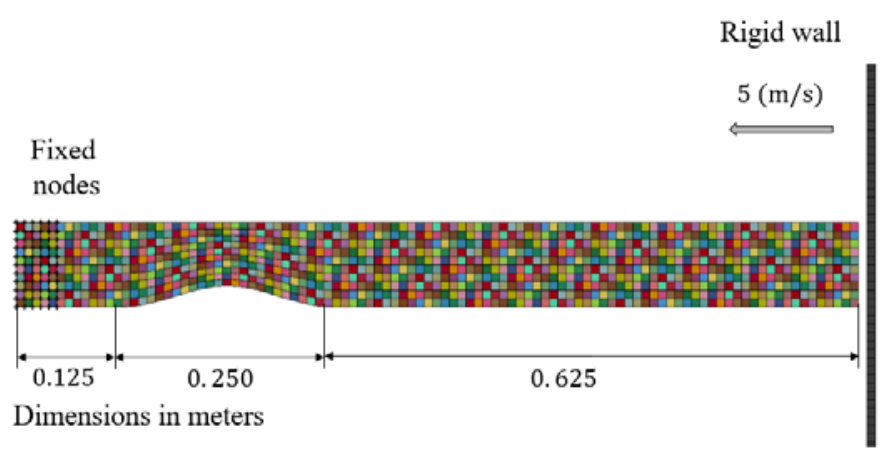

Figure 4. Geometry and finite element model of the square tube.

The initial design (uniform thickness of $5 \mathrm{~mm}$ ) collapses globally at the geometric imperfection (Figure 11.a). The proposed modified HCA method continuously changes the thickness distribution in order to move the zones with high IED from the geometric imperfection to the end of the tube that receives the impact. Figure 5 is a comparison of the IED distribution at iterations 1,3 , and 5 . Figure 6 shows the change in the thickness distribution of the square tube at iterations 1,3 and 5 .
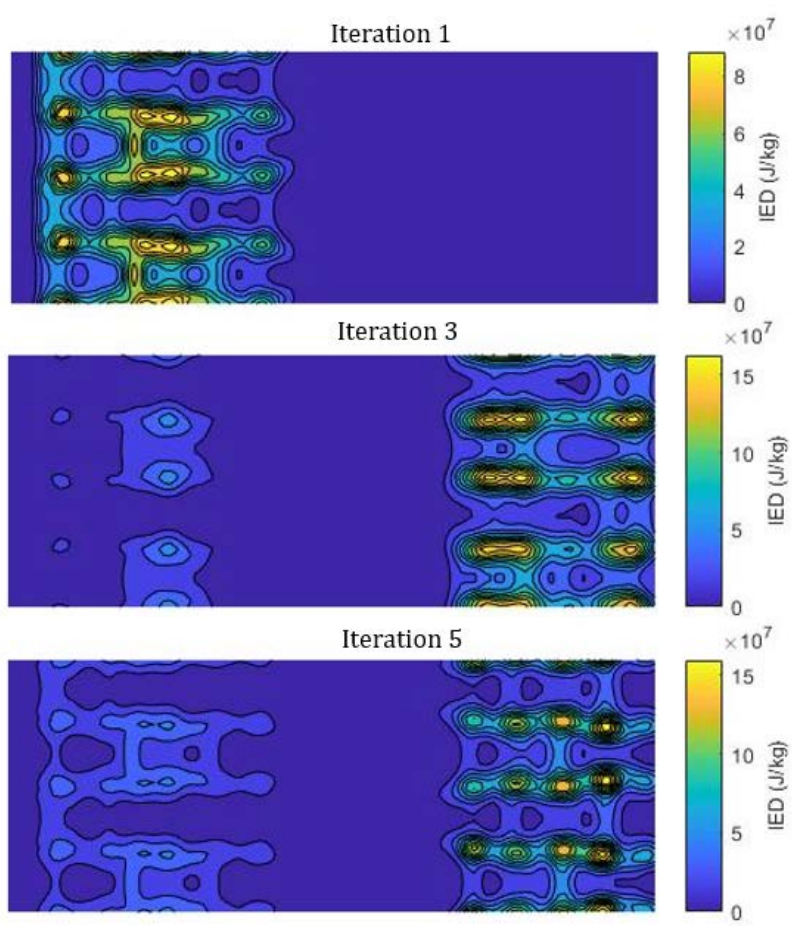

Figure 5. IED distribution of the square tube designs at iterations 1, 3 and 5. 
Iteration 1

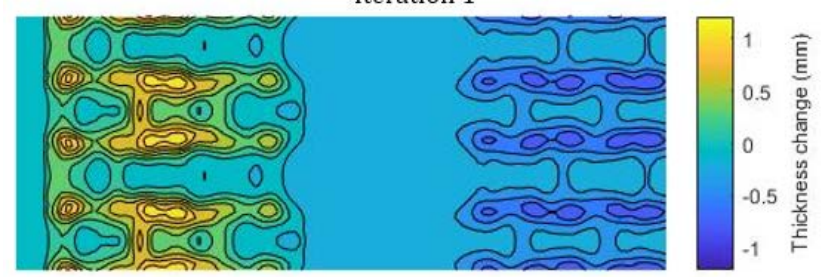

Iteration 3

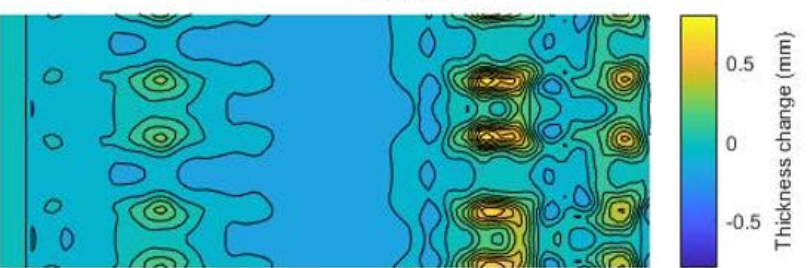

Iteration 5

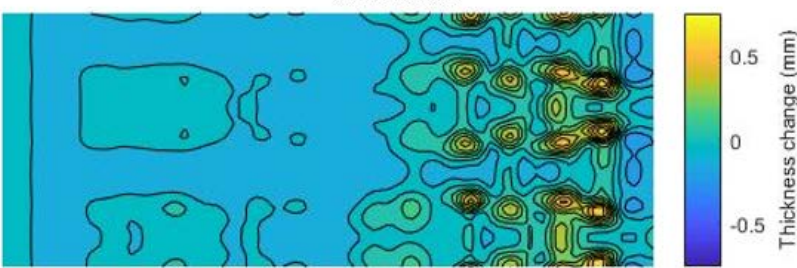

Figure 6. Change in thickness distribution of the square tube designs at iterations 1,3 and 5 .

The proposed methodology find designs with a larger area under the force-displacement curve without significantly affecting the PCF as illustrated in Figure 7. The energy absorbed by designs at iterations 3 and 5 is higher than the initial design.

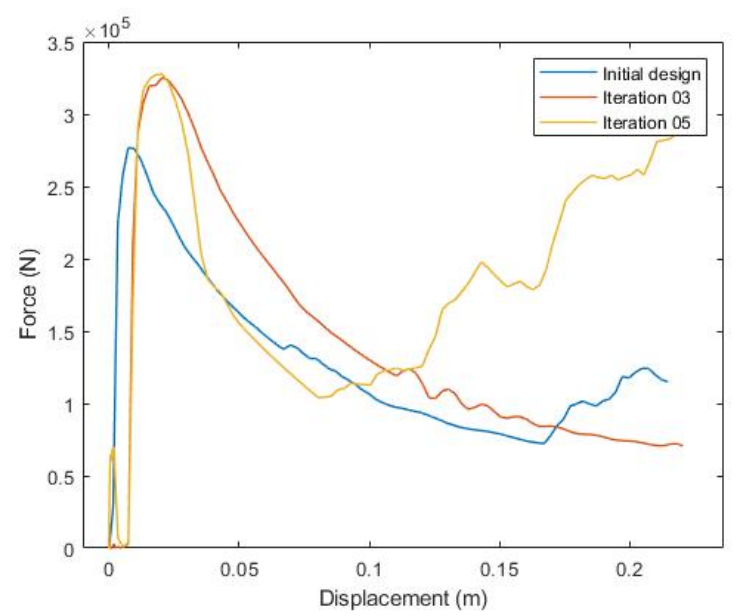

Figure 7. Force - displacement curves of the square tube designs at iterations 1,3 and 5.

When the weight factor $\mathrm{w}$ of the prescribed field is 0.50 , the algorithm presents an oscillatory convergence (Figure 8). Therefore, at the end of the searching process, a Pareto front in terms of SEA and PCF is generated (Figure 9). One of the members of the Pareto optimal set is found at iteration 52 whose thickness distribution is shown in Figure 10. The design of iteration 52 shows that it is not necessary to reinforced the entire surface of the geometric imperfection in order to prevent global collapse. Figure 11 compares the collapse of the initial square tube and the optimized designs at iteration 52, which feature progressive collapse. The percent change in SEA and PCF, with respect to the initial design, is summarized in Table 2 . The algorithm found designs that improve the SEA by $16 \%$ to $64 \%$. No design had an increment in the PCF larger than $7 \%$.

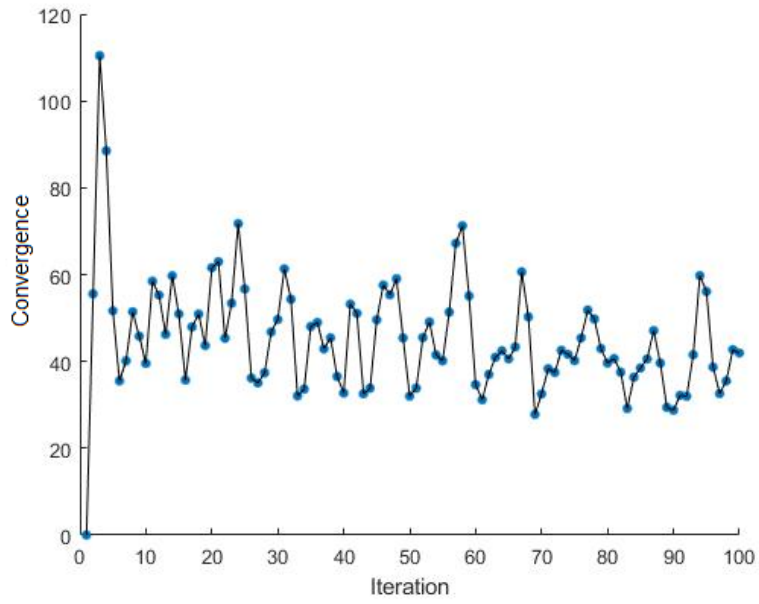

Figure 8. Convergence curve of the square tube problem using a weight factor of 0.50 .

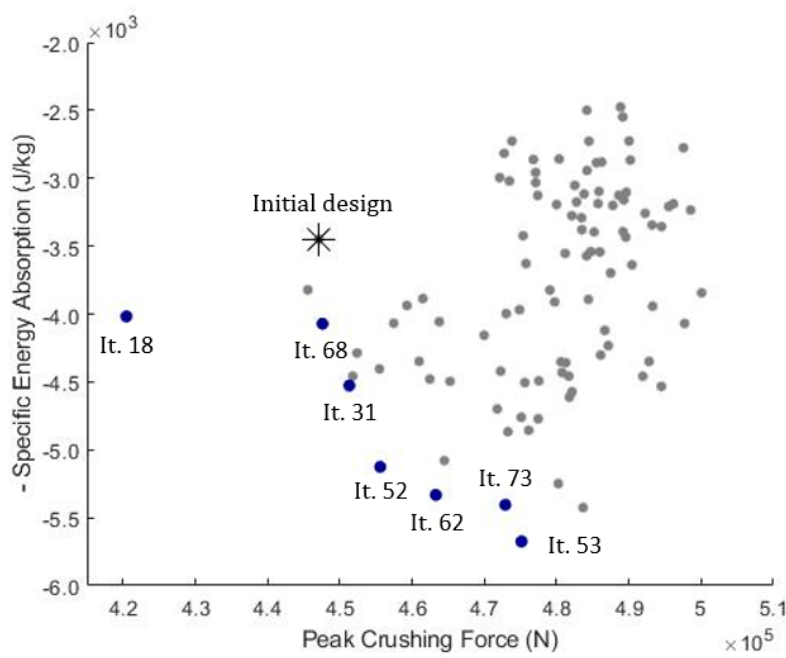

Figure 9. SEA and PCF of optimized square tube designs using a weigh factor of 0.50 . The Pareto front is shown with blue dots.

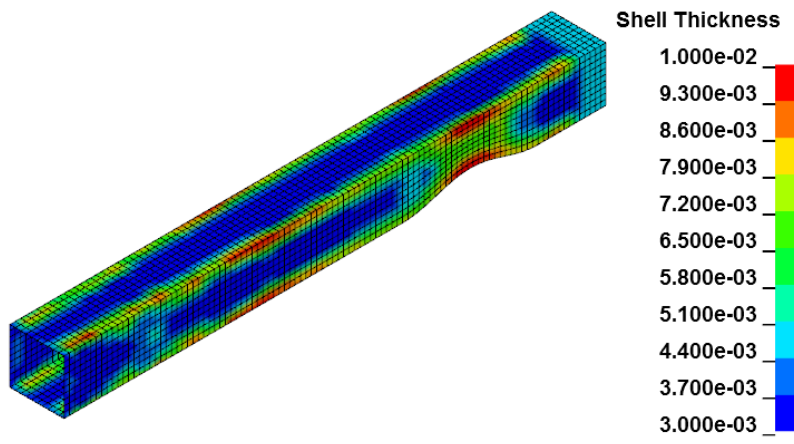

Figure 10. Thickness distribution of the square tube at iteration 52 using a weight factor of 0.50 .

Page 6 of 10 

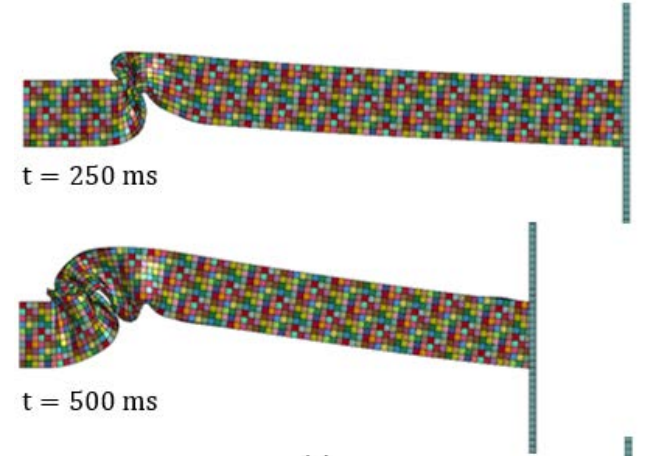

(a)
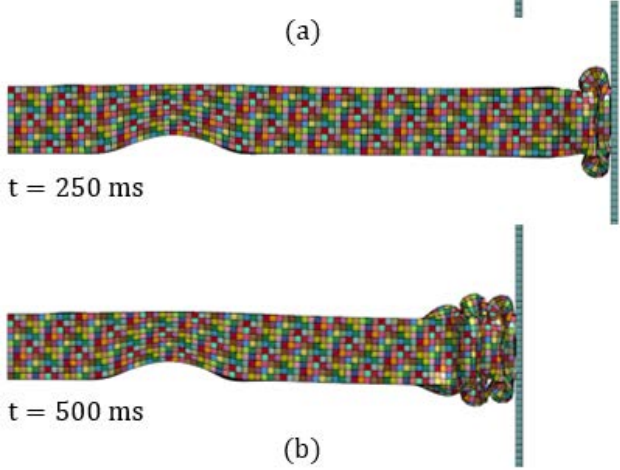

(b)

Figure 11. Collapse comparison of the (a) uniform thickness and (b) iteration 52 square tube designs using a weight factor of 0.50 .

Table 2. Change in SEA and PCF (with respect to the initial design) of the Pareto front square tube designs; using a weight factor of 0.50 .

\begin{tabular}{|c|c|c|}
\hline Iteration & $\Delta$ SEA (\%) & $\Delta$ PCF (\%) \\
\hline 18 & 16.47 & -5.97 \\
\hline 68 & 18.05 & 0.11 \\
\hline 31 & 31.26 & 0.94 \\
\hline 52 & 48.60 & 1.89 \\
\hline 62 & 54.60 & 3.62 \\
\hline 73 & 56.71 & 5.77 \\
\hline 53 & 64.55 & 6.27 \\
\hline
\end{tabular}

\section{S-Rail}

The second example is an S-rail with a geometric imperfection. The finite element model has a mesh size of $40 \times 102$ with an average element size of $0.01 \times 0.01 \mathrm{~m}$. The S-rail has a square cross-section of size $0.1 \mathrm{~m}$. In the FEA model, the nodes of one end of the S-rail are fixed, while the other end is axially impacted by a rigid plate with a prescribed constant velocity of $5 \mathrm{~m} / \mathrm{s}$ (Figure 12). Similar to the square tube example, the thickness of each shell element can range from 3 to $10 \mathrm{~mm}$. The proposed methodology is used to find the thickness distribution that will lead to progressive collapse when using a mass fraction of 0.5. In order to assess the effect of the weight factor w of the prescribed field in the final designs, values of $0.25,0.75$ and 1.25 are used. The simulation time is $500 \mathrm{~ms}$.

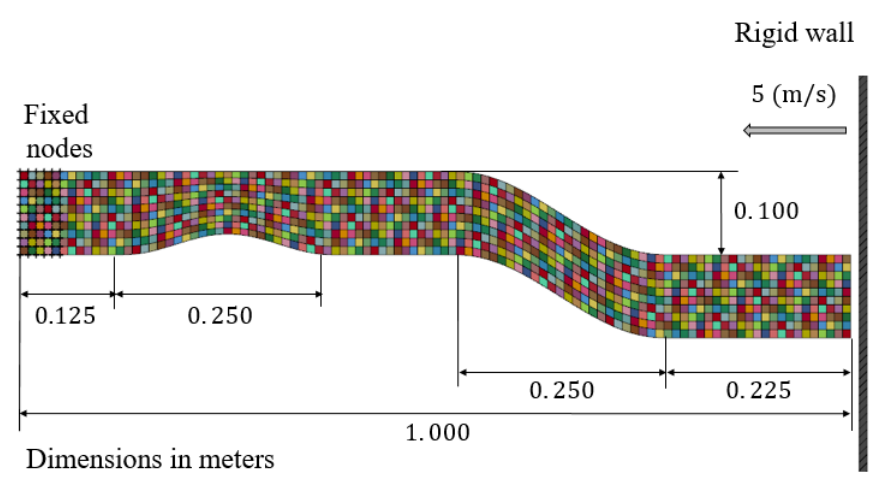

Figure 12. Geometry and finite element model of the S-rail.

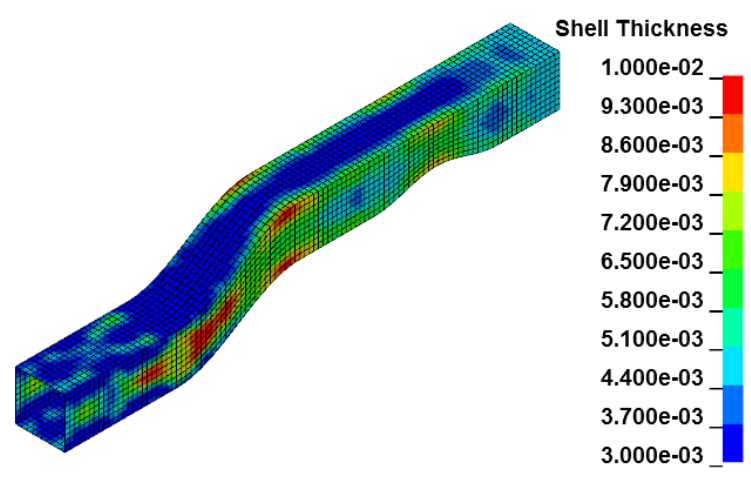

Figure 13. Thickness distribution of the S-rail at iteration 44 using a weight factor of 0.75 .
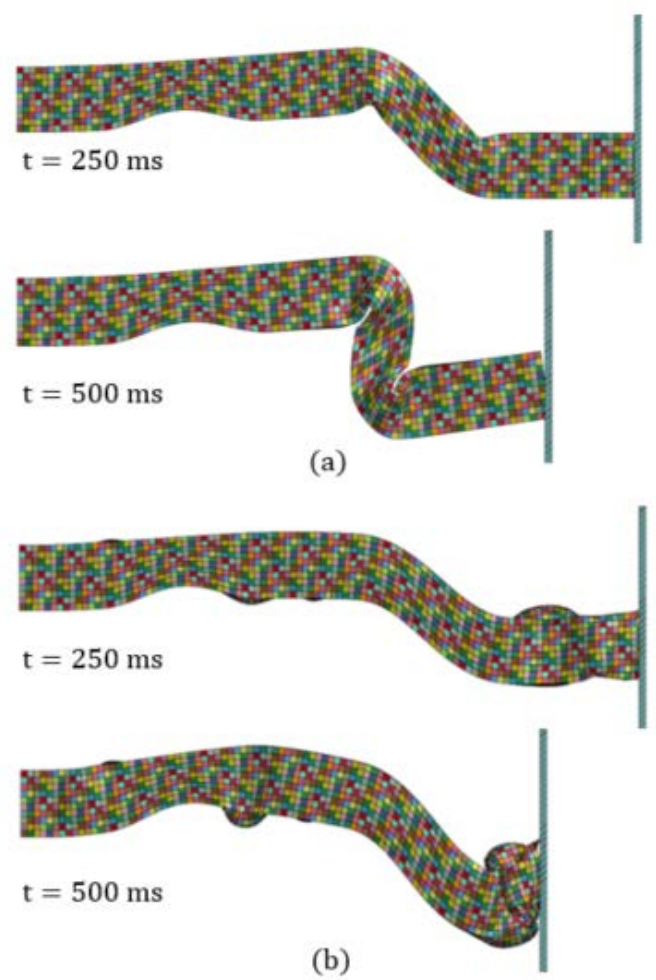

Figure 14. Collapse comparison of the (a) uniform thickness and (b) iteration 44 S-rail designs using a weight factor of 0.75 . 
Figure 13 shows the thickness distribution of an S-rail design at iteration 44 using a w of 0.75 . Figure 14 compares the collapse of this design and the initial design (uniform thickness of $5 \mathrm{~mm}$ ). Due to geometrical complexity of the S-rail, the initial design undergoes global collapse (Figure 14.a) while the thickness distribution of the Srail at iteration 44 initiates progressive collapse (Figure 14.b). The design at iteration 44 presents larger values of PCF and energy absorption than the corresponding values of the initial design (Figure 15). An increment in the PCF of $23 \%$ represents an increase of $61 \%$ in the energy absorption.

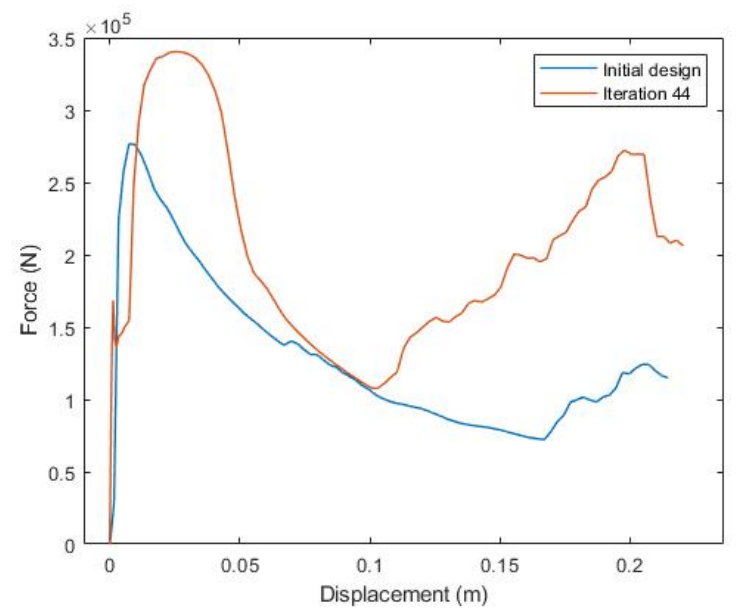

Figure 15. Force - displacement curves of the S-rail designs at iterations 1 and 44 ; using a weight factor of 0.75 .

The weight factor $\mathrm{w}$ of the prescribed response field determines the crashworthiness of the optimized designs and the convergence of the algorithm. In terms of the convergence and stability of the algorithm, a faster and more stable convergence is achieved when using large $\mathrm{w}$ factors (Figure 16). From a crashworthiness standpoint, the change in SEA and the PCF is inversely proportional to the $w$ factor (Figure 17). For example, the designs generated with the smallest weight factor $(\mathrm{w}=0.25)$ exhibit the highest SEA and PCF values. Each weight factor generates a different Pareto front as shown in Figure 17. Some examples of the S-rail designs that are members of these Pareto fronts are shown in Figure 18.

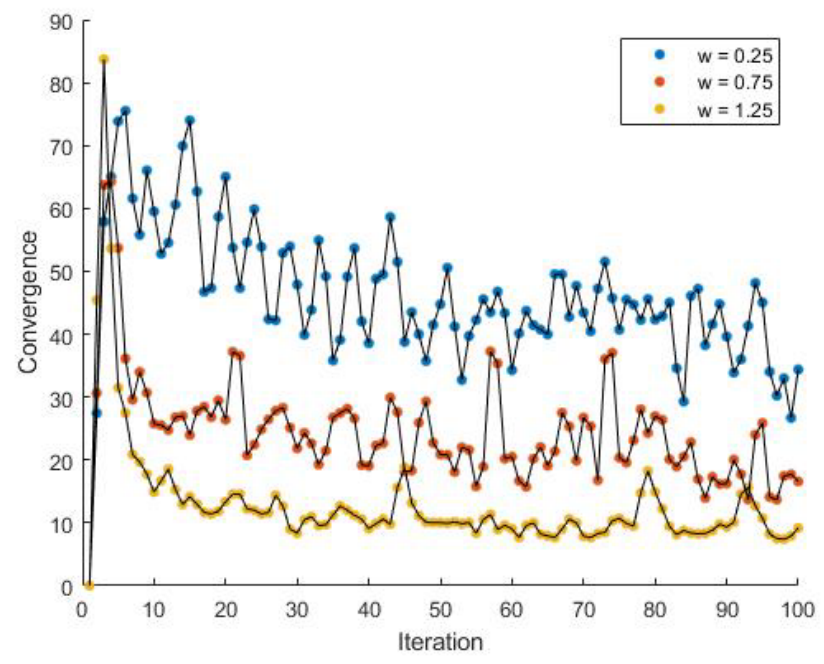

Figure 16. Convergence curves of the S-rail problem using weight factors of $0.25,0.75$ and 1.25 .

Using as a reference the SEA and PCF of the initial design, the modified HCA method finds designs that increase the SEA within a range of $38 \%$ to $102 \%$ with increments in the PCF up to $21 \%$ (Table 3).

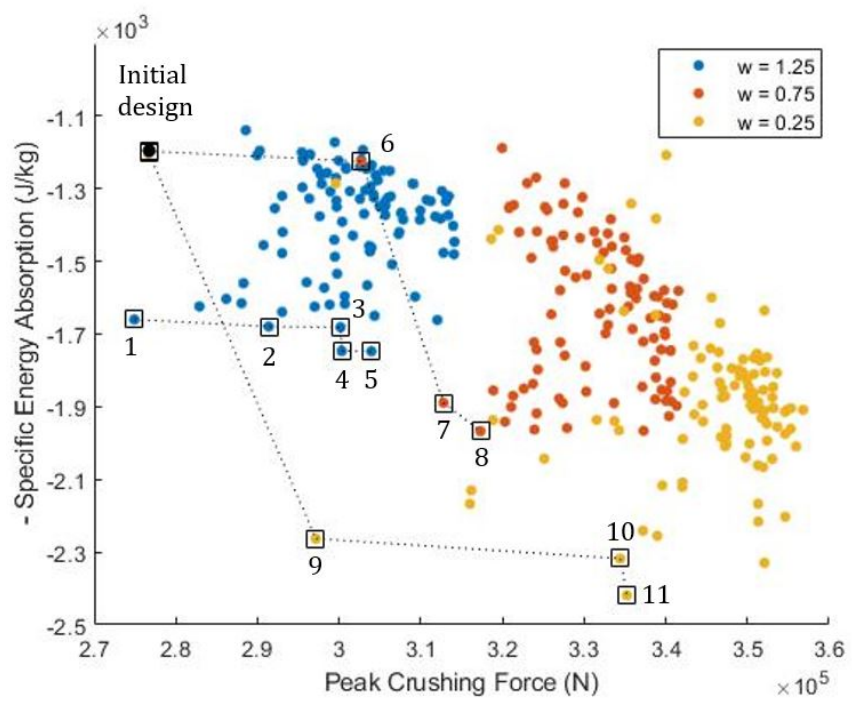

Figure 17. SEA and PCF of optimized S-rail designs and Pareto fronts using weight factors of $0.25,0.75$ and 1.25 . 


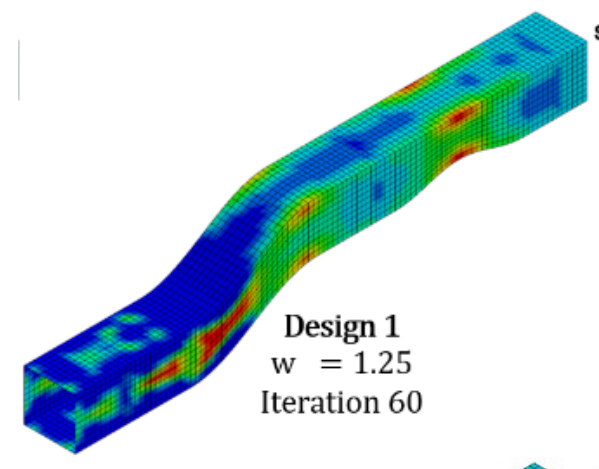

hell Thickness

$1.000 \mathrm{e}-02$

$9.300 \mathrm{e}-03$

$8.600 \mathrm{e}-03$

$7.900 \mathrm{e}-03$

$7.200 \mathrm{e}-03$

$6.500 \mathrm{e}-03$

$5.800 \mathrm{e}-03$

$5.100 \mathrm{e}-03$

$4.400 \mathrm{e}-03$

$3.700 \mathrm{e}-03$

$3.000 \mathrm{e}-03$

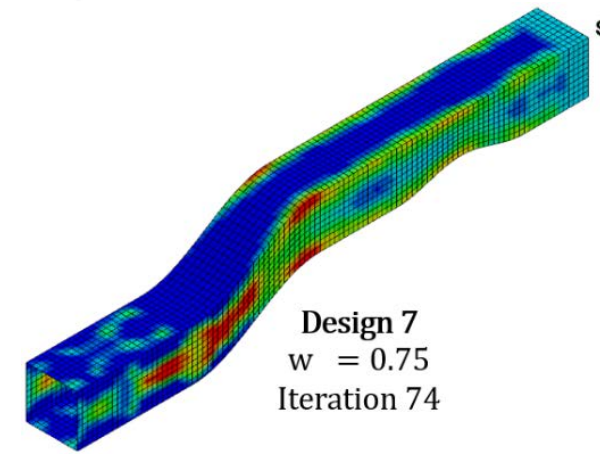

Shell Thickness

$1.000 \mathrm{e}-02$

$9.300 \mathrm{e}-03$

$8.600 \mathrm{e}-03$

$7.900 \mathrm{e}-03$

$7.200 \mathrm{e}-03$

$6.500 \mathrm{e}-03$

$5.800 \mathrm{e}-03$

$5.100 \mathrm{e}-03$

$4.400 \mathrm{e}-03$

$3.700 \mathrm{e}-03$

$3.000 \mathrm{e}-03$

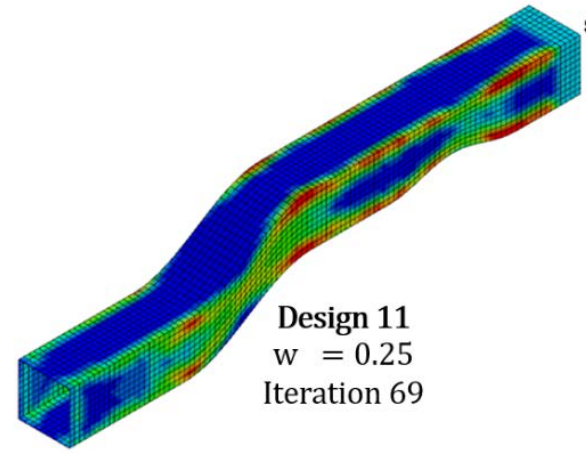

Shell Thickness

1.000e-02

$9.300 \mathrm{e}-03$

$8.600 \mathrm{e}-03$

$7.900 \mathrm{e}-03$

$7.200 \mathrm{e}-03$

$6.500 \mathrm{e}-03$

$5.800 \mathrm{e}-03$

$5.100 \mathrm{e}-03$

$4.400 \mathrm{e}-03$

$3.700 \mathrm{e}-03$

$3.000 \mathrm{e}-03$

Figure 18. Thickness distribution of select Pareto front S-rails using weight factors of $0.25,0.75$ and 1.25 .

Table 3. Change in SEA and PCF (with respect to the initial design) of the Pareto front S-rail designs; using weight factors of $0.25,0.75$ and 1.25

\begin{tabular}{|c|c|c|c|c|}
\hline Design & $\mathbf{w}$ & Iteration & $\Delta$ SEA $(\%)$ & $\triangle \mathrm{PCF}(\%)$ \\
\hline 1 & \multirow{5}{*}{1.25} & 60 & 38.85 & -0.65 \\
\hline 2 & & 66 & 40.47 & 5.30 \\
\hline 3 & & 82 & 40.62 & 8.48 \\
\hline 4 & & 86 & 46.10 & 8.53 \\
\hline 5 & & 97 & 46.16 & 9.84 \\
\hline 6 & \multirow{3}{*}{0.75} & 2 & 2.42 & 9.39 \\
\hline 7 & & 74 & 58.03 & 13.04 \\
\hline 8 & & 80 & 64.55 & 14.67 \\
\hline 9 & \multirow{3}{*}{0.25} & 95 & 89.29 & 7.38 \\
\hline 10 & & 85 & 93.89 & 20.85 \\
\hline 11 & & 69 & 102.35 & 21.15 \\
\hline
\end{tabular}

4. Alexander, J.M., An approximate analysis of the collapse of thin cylindrical shells under axial loading. The Quarterly Journal of Mechanics and Applied Mathematics, 1960. 13(1): p. 10-15.

5. Wierzbicki, T. and W. Abramowicz, On the crushing mechanics of thin-walled structures. Transactions of the ASME. Journal of Applied Mechanics, 1983. 50(4A): p. 727-34.

6. Abramowicz, W. and N. Jones, Dynamic axial crushing of square tubes. International Journal of Impact Engineering, 1984. 2(2): p. 179-208.

7. Abramowicz, W. and N. Jones, Dynamic axial crushing of circular tubes. International Journal of Impact Engineering, 1984. 2(3): p. 263-281.

8. Abramowicz, W. and N. Jones, Dynamic progressive buckling of circular and square tubes. International Journal of Impact Engineering, 1986. 4(4): p. 243-270. 
9. Langseth, M., O.S. Hopperstad, and A.G. Hanssen, Crash behaviour of thin-walled aluminium members. Thin-Walled Structures, 1998. 32(1): p. 127-150.

10. Fyllingen, O., et al., Modelling of tubes subjected to axial crushing. Thin-Walled Structures, 2010. 48(2): p. 134-142.

11. Tarigopula, V., et al. Axial crushing of thin-walled high-strength steel sections. 2006. Elsevier Ltd.

12. Chung Kim Yuen, S. and G.N. Nurick, The energyabsorbing characteristics of tubular structures with geometric and material modifications: an overview. Applied Mechanics Review, 2008. 61(2): p. 1-15.

13. Baroutaji, A., M. Sajjia, and A.-G. Olabi, On the crashworthiness performance of thin-walled energy absorbers: Recent advances and future developments. Thin-Walled Structures, 2017. 118: p. 137-163.

14. Shinde, S., et al., Structural Optimization of ThinWalled Tubular Structures for Progressive Buckling Using Compliant Mechanism Approach. SAE International Journal of Passenger Cars - Mechanical Systems, 2013. 6(1): p. 109-120.

15. Fang, H., et al., A comparative study of metamodeling methods for multiobjective crashworthiness optimization. Computers \& Structures, 2005. 83(25): p. 2121-2136.

16. Fang, J., et al., On design optimization for structural crashworthiness and its state of the art. Structural and Multidisciplinary Optimization, 2017. 55(3): p. 10911119.

17. Tovar, A., et al., Topology optimization using a hybrid cellular automation method with local control rules. Journal of Mechanical Design, Transactions of the ASME, 2006. 128(6): p. 1205-1216.
18. Patel, N.M., et al., Crashworthiness Design Using Topology Optimization. Journal of Mechanical Design, 2009. 131(6): p. 061013-061013-12.

19. Shinde, S., D. Detwiler, and A. Tovar. Structural Optimization of Thin-Walled Tubular Structures using Weighted Multi-Objective Approach. in 10th World Congress on Structural and Multidisciplinary Optimization. 2013.

20. Bandi, P., A. Tovar, and J.E. Renaud. Design of 2D and $3 D$ non-linear compliant mechanisms using hybrid cellular automata. in 52nd AIAA/ASME/ASCE/AHS/ASC Structures, Structural Dynamics and Materials Conference, April 4, 2011 April 7, 2011. 2011. Denver, CO, United states: American Institute of Aeronautics and Astronautics Inc.

21. Hallquist, J.O., LS-DYNA theory manual. Livermore software technology corporation, 2006. 3.

\section{Contact Information}

Homero Valladares, Ph.D. Student in Mechanical Engineering Phone no: +1 (317) 748-4039

hvallada@purdue.edu

Joel Najmon, Ph.D. Student in Mechanical Engineering

Phone no: +1 (317) 627-2393

jnajmon@purdue.edu

Andres Tovar, Ph.D., Associate Professor of Mechanical and Energy Engineering

Indiana University, Purdue University - Indianapolis

Phone no: +1 (317) 278-7090

tovara@iupui.edu 